\title{
Design of Multiplier-Less MDFT Filter Banks with Perfect Reconstruction using ABC Algorithm
}

\author{
V. Sakthivel \\ Department of Electronics and \\ Communication Engineering \\ National Institute of Technology \\ Calicut Kerala 673601, India
}

\author{
Rathlavath Chandru \\ Department of Electronics and \\ Communication Engineering \\ National Institute of Technology \\ Calicut Kerala 673601, India
}

\author{
Elizabeth Elias \\ Department of Electronics and \\ Communication Engineering \\ National Institute of Technology \\ Calicut Kerala 673601, India
}

\begin{abstract}
In this paper the design of a totally multiplier-less M-Channel Modified Discrete Fourier Transform (MDFT) Filter bank with Perfect reconstruction has been proposed. Canonic Sign Digit (CSD) based Finite Impulse Response (FIR) prototype filter with low implementation complexity is designed. The performance of the MDFT filter bank designed with this filter is optimized using Artificial Bee Colony (ABC) algorithm. This design leads to very low implementation complexity and hence low power dissipation and low chip area, which are desirable in upcoming applications such as software defined radio, wireless communication and portable computing systems.
\end{abstract}

\section{General Terms}

Multiplier-less Modified DFT filter, CSD, Optimization

\section{Keywords}

Multiplier-less Modified DFT filter banks with Perfect Reconstruction, Canonic Signed Digit, Artificial Bee Colony Algorithm

\section{INTRODUCTION}

Filter banks are used in many digital signal processing applications such as sub-band coding and multiple carrier data transmission. There are different filter bank structures available, but the modulated filter banks are very famous since their design is simple and are easy to implement. There are various modulated filter banks, among them the Discrete Fourier Transform (DFT) polyphase filter bank [1] provides the highest computational efficiency, in some cases, higher than that of the Cosine modulated filter bank, but it is not having inherent alias cancellation structure. For M-channel perfect reconstruction uniform filter banks, the polyphase matrix of the analysis and synthesis filters should be invertible. The modified DFT filter banks [2 - 4] can be used to nullify the disadvantage of the DFT filter banks. MDFT filter banks are modified complex modulated, critically subsampled filter banks based on the DFT filter banks. The analysis and synthesis filters have linear phase. All odd alias spectra can be automatically cancelled using the structure which results in Near Perfect Reconstruction (NPR) MDFT filter banks. Here, the aliasing distortion and amplitude distortion are kept very small. PR filter banks are required in many applications such as image processing. Hence it is desirable to go for MDFT filter bank with PR.

The output signals in the MDFT filter banks and Noncritically decimated DFT filter banks differ only in a scaling factor and an additional time delay [5]. So it is sufficient to consider the non-critically sub-sampled DFT filter banks for perfect reconstruction (PR). This will simplify the structure for PR [5]. To achieve PR, there should be exact compensation of aliasing and no amplitude and phase distortion. There will be no phase distortions in MDFT filter banks since all the filters are chosen to have linear phase. The polyphase realization of the DFT filter banks will be useful to get PR in analysis and synthesis filters [5].

The implementation complexity can be significantly reduced when the continuous filter coefficients are represented in the Signed Power of Two (SPT) space [6]. The multiplications in filter implementation will be replaced by shifters and adders in the SPT space. This will lead to totally multiplier-less MDFT filter banks with PR. This will reduce the implementation complexity, power consumption and chip area [2]. Canonic Signed Digit (CSD) representation is a special case of the SPT space, which uses both additions and subtractions [7]. Due to this, the coefficients can be represented using minimum number of non-zero SPT terms. The performance of the filter degrades when the continuous filter coefficients are represented as discrete filter coefficients using CSD space. Hence, to improve the performance of the CSD represented filter coefficients, efficient non-linear optimization techniques have to be deployed in the discrete space. Since the search space contains integers, classical gradient based optimization technique cannot be used. In such cases, Meta-heuristic algorithm is a good choice [8]. In this paper, artificial bee colony (ABC) algorithm [9 - 12] is used for the design of the optimal multiplier-less FIR prototype filter and hence the MDFT filter banks with PR. Such a multiplier-less MDFT filter bank with PR is not proposed in the literature so far.

The rest of the paper is organized as given below. In Section 2, an overview of MDFT filter banks is presented. Section 3 describes the MDFT filter banks with PR. Section 4 describes the CSD representation. Section 5 gives details about ABC optimization algorithm. Section 6 shows the proposed design of the multiplier-less MDFT filter banks with PR, Section 7 gives the simulation results and discussions. Section 8 concludes the paper.

\section{OVERVIEW OF MDFT FILTER BANKS}

The structure of the DFT filter bank is shown in Fig. 1 in which $\mathrm{H}_{\mathrm{k}}(\mathrm{z})$ and $\mathrm{F}_{\mathrm{k}}(\mathrm{z})$ represent the analysis and synthesis filters respectively and $\uparrow \mathrm{M}$ and $\downarrow \mathrm{M}$ represent interpolation and decimation by $M$ respectively. Here, the analysis and synthesis filters are derived by complex modulation from a linear phase FIR prototype filter $\mathrm{H}(\mathrm{z})$ with a transition band from $-\pi / M$ to $\pi / M$. The analysis filters are represented as [13]

$H_{k}(z)=H\left(z W_{M}^{k}\right)$ 
The synthesis filters are defined as [13]

$F_{k}(z)=M H\left(z W_{M}^{k}\right)$

where $\quad W_{M}=\exp \left[-j\left(\frac{2 \pi}{\boldsymbol{M}}\right)\right]$

The reconstructed signal at the output of the filter banks is defined as [13]

$\widehat{X}(z)=\frac{1}{M} \sum_{k=0}^{M-1} F_{k}(z) \sum_{l=0}^{M-1} H\left(z W_{M}^{k+1}\right) X\left(z W_{M}^{l}\right)$

assuming $X(z)$ is the input signal.

Since the prototype filter is band-limited to $2 \pi / \mathrm{M}$, all the nonadjacent alias components are removed. If we consider only adjacent alias components, the reconstructed signal can be written as [13]

$\hat{X}(z)=\frac{1}{M} \sum_{k=0}^{M-1} F_{k}(z) \sum_{l=-1}^{1} H\left(z W_{M}^{k+1}\right) X\left(z W_{M}^{l}\right)$

From equation 4, we can understand that the reconstructed signal contains alias components.

There is no way in the structure of the DFT filter banks to compensate the alias signals.

Hence perfect reconstruction is not possible in DFT filter banks due to aliasing and linear distortion. To overcome this disadvantage some modifications have been done in the DFT filter banks, which result in the MDFT filter banks [5].

The MDFT filter bank is a complex modulated M-channel filter bank, with a two step decimation of the sub-band signals. Initially the sampling rate is decimated by a factor $\mathrm{M} / 2$ and later decimated by the factor 2 with and without a delay of one sampling period, using either the real or the imaginary part in the sub-bands respectively as shown in Fig.2 [5]. All adjacent alias spectra and all odd alias spectra are cancelled within the MDFT filter banks due to the modifications in the structure of the filter banks.

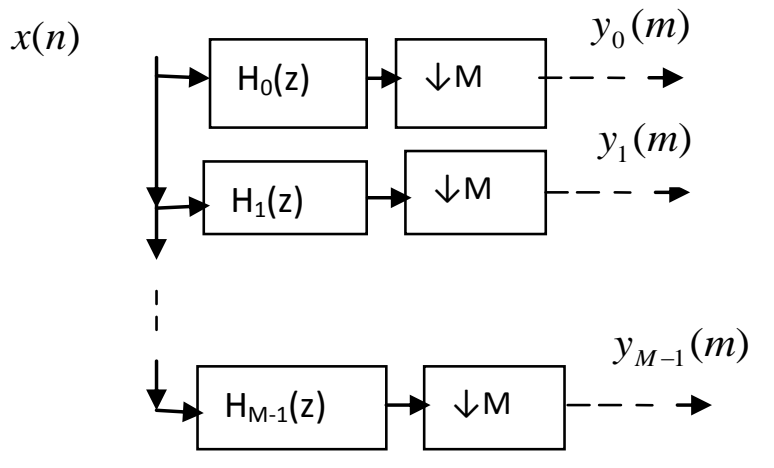

Fig.1.The structure of DFT filter bank
But in applications like image processing, PR filter banks are preferred. Hence we need to design the MDFT filter banks with PR.

\section{MDFT FILTER BANKS WITH PR}

If $X(z)$ is the input signal for MDFT filter banks as shown in Fig.2, the reconstructed signal can be written as [5]

$\hat{X}(z)=\frac{z^{-M / 2}}{M} \sum_{k=0}^{M-1} \sum_{l=0}^{\frac{M}{2}-1} F_{k}(z) H_{k}\left(z W_{M}^{2 l}\right) X\left(z W_{M}^{2 l}\right)$

From equation 5 , we can see that the reconstructed signal contains only even alias spectra and all odd aliasing terms are automatically cancelled.

The reconstructed signal from a non-critically decimated DFT filter bank with M-channels and a sampling rate decimation of $\mathrm{M} / 2$ as shown in Fig.3, can be written as [5]

$$
\hat{X}_{D F T}(z)=\frac{2}{M} \sum_{k=0}^{M-1} \sum_{l=0}^{\frac{M}{2}-1} F_{k}(z) H_{k}\left(z W_{M}^{2 l}\right) X\left(z W_{M}^{2 l}\right)
$$

The reconstructed signals $\hat{X}(z)$ and $\hat{X}_{D F T}(z)$ are the same but only differ in the delay and the scaling factor of the amplitude.

Therefore, for PR, it is enough to consider the non-critically sub-sampled DFT filter bank as shown in Fig. 3 instead of the MDFT filter banks [5]. By considering like this, to get PR it is not required to take the real and imaginary parts respectively in the subbands of the structure in the MDFT filter banks.

For perfect reconstruction, the filter bank should have:

I. Exact compensation of aliasing
II. No phase and amplitude distortion

To implement the PR condition on the analysis and synthesis filters of Fig.3, the polyphase realization of the DFT filter banks is needed.

In the analysis filter bank, type-1 polyphase filters of the low pass filter $h_{o}(n)$ are used [13, 14]. The polyphase components are given by

$g_{k}(m)=h_{o}(m \cdot M+k), \quad k=0,1,2, \ldots \ldots . M-1$
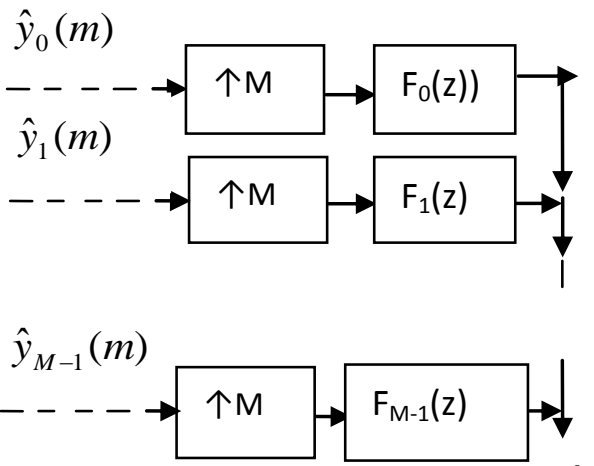

$\hat{x}(n)$

Prototype filter can be designed with high stop-band attenuation to keep the non adjacent alias terms very small. This will give near perfect reconstruction (NPR) of the input signals in MDFT filter banks. 
$X(z)$
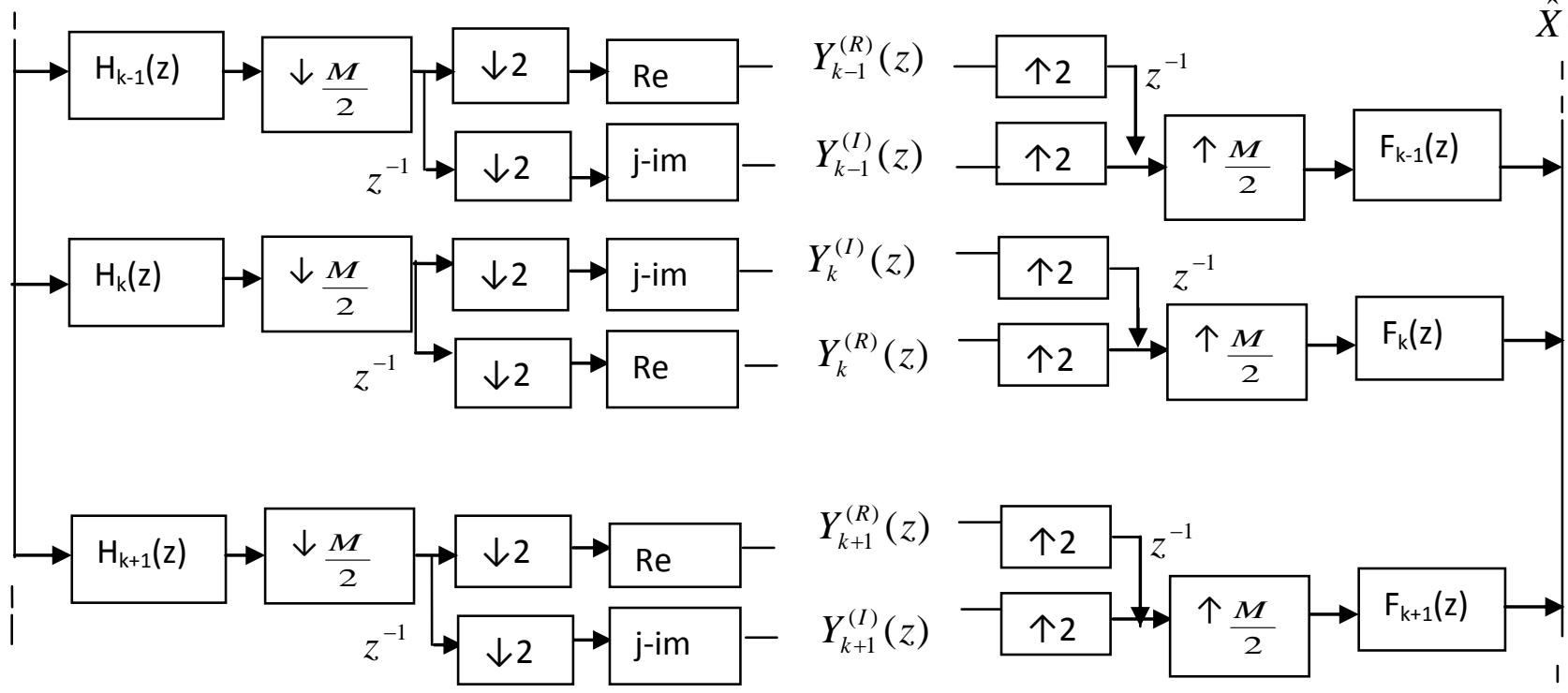

Fig.2. Modified DFT filter bank

In the synthesis filter bank, type-3 polyphase filters are used $[13,14]$.

$\overline{g_{k}}(m)=h_{o}(m . M-k), \quad k=0,1,2, \ldots \ldots \ldots \ldots M-1$

It is proved in [5] that if the prototype filter has a length of $N=r . M+1$, where $\mathrm{r}$ is an integer, then the MDFT filter banks guarantee PR if the following constraints of the polyphase filters are satisfied [5].

$G_{k}(z) \bar{G}_{k}(z)+G_{k+\frac{M}{2}}(z) \bar{G}_{k+\frac{M}{2}}(z)=\frac{2}{M} z^{-\alpha}$

$k=0,1 \ldots \ldots \ldots \frac{M}{2}-1$, where $\alpha$ is a delay

For the general case, $N=r . M+s$

with $r \in$ Integer and $0 \leq s<M$,

Then the MDFT filter banks guarantee PR if the following constraints of the polyphase filters are satisfied [5].

$G_{k}(z) \tilde{G}_{k}(z)+G_{k+\frac{M}{2}}(z) \tilde{G}_{k+\frac{M}{2}}(z)=\frac{2}{M} \Rightarrow \mathrm{p}(\mathrm{z})$

Amplitude distortion function of the MDFT filter bank can be obtained as $[13,15]$

$T_{\text {dist }}(z)=\frac{1}{M} \sum_{k=0}^{M-1} F_{k}(z) H_{k}(z)$

In the MDFT filter bank, the PR condition can be obtained if equation 9 or 10 is satisfied depending on the value of $s$.

\section{CANONIC SIGNED DIGIT (CSD) REPRESENTATION}

If the filter coefficients are represented in the signed power of two (SPT) form, the circuit complexity can be reduced. Among the various forms, canonic signed digit (CSD) representation is a minimal one. The CSD represented filter offers minimum implementation complexity, power consumption and chip area compared to other representations. CSD is a number system for encoding a floating-point value in a two's complement representation. This encoding contains fewer non-zeros than the two's complement form, leading to efficient implementations of add/subtract networks in Digital Signal Processing (DSP) hardware. Canonic signed digit is based on the ternary number system $(-1,0$ and 1$)$. It is a unique representation of a binary number with minimum number of 1 and -1 digits [6,7]. The number of non-zero bits in the CSD expression is equal to the number of signed power of two terms (SPT). The number of adders/subtractors and shifters can be reduced by converting the filter coefficients to CSD representation. Hence the filter bank can be implemented without multipliers.

A fractional number $\mathrm{g}$ is represented in CSD format as [7]

$g=\sum_{j=1}^{P} d_{j} 2^{R-j}$

where $\mathrm{P}$ is the word length of the CSD number,

$d_{j}=\{-1,0,1\}$ and the integer $\mathrm{R}$ represents a radix point in the range $0<\mathrm{R}<\mathrm{P}$

The following are the properties of CSD numbers:

- No two consecutive bits in a CSD number are nonzero.

- The CSD representation of a number contains the minimum possible number of non-zero bits. 


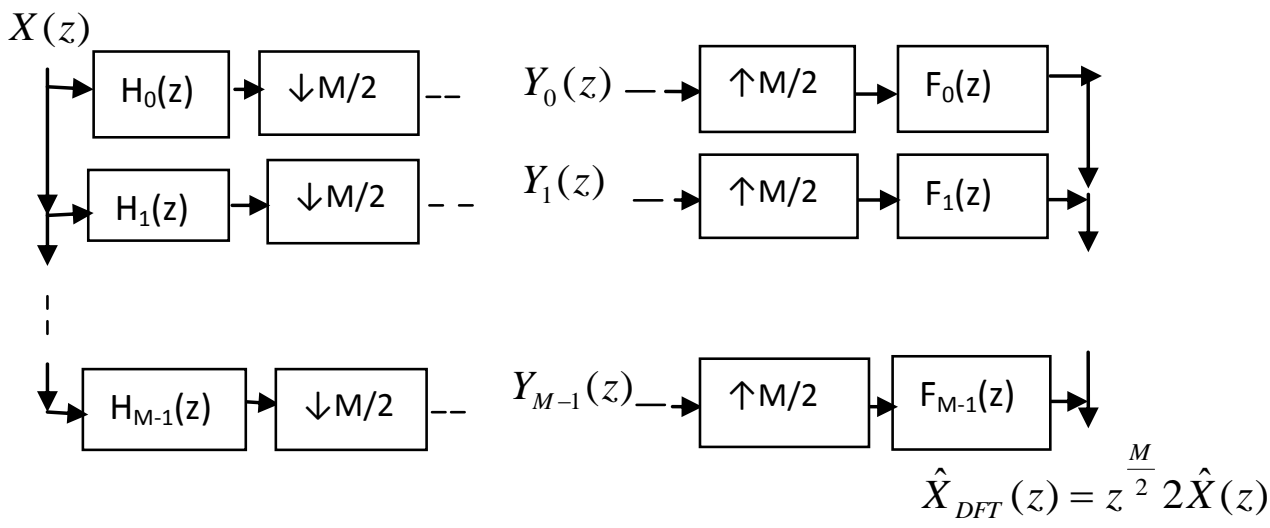

Fig.3. Complex modulated M-channel filter bank with a sub-sampling factor of $M / 2$

An $n$ bit CSD number does not have more than $(n+1) / 2$ nonzero bits, often fewer.

A look-up-table approach can be used for the fast conversion of the filter coefficients to their CSD equivalents [16]. A typical look-up table entry is shown in Table.1. But the CSD representations of the coefficients may degrade the filter performances. Hence some optimization techniques are to be deployed to improve the filter performances. In this paper the artificial bee colony algorithm is used to improve the performances.

Table.1. 16 bit CSD look-up-table

\begin{tabular}{|c|c|c|c|}
\hline Index & CSD Equivalent & $\begin{array}{c}\text { Decimal } \\
\text { Equivalent }\end{array}$ & $\begin{array}{c}\text { Number of } \\
\text { Non-zeros }\end{array}$ \\
\hline 43691 & 0101010101010101 & 0.6667 & 8 \\
\hline
\end{tabular}

\section{ARTIFICIAL BEE COLONY (ABC) ALGORITHM}

An efficient algorithm for optimization is introduced by Karaboga and basturk called Artificial Bee Colony (ABC) algorithm $[9-12,17]$. This algorithm contains employed bees, onlooker bees and scout bees. Employed bees will find a food source within the neighborhood of the food source in their memory. Onlooker bees will wait on the dance area to collect information from employed bees to select a food source. Scout bees will search randomly the environment surrounding the nest for new food sources. The possible solution to a problem to be optimized is the position of the food source.

The quality of the solution represented by the food source is based on the amount of nectar of that food source. The nectar quality of a food source represents the fitness value of the corresponding solution.

Integer coded $\mathrm{ABC}$ algorithm has been proposed for the design of non-uniform filters bank transmultiplexer in [16]. In this paper, the integer coded $\mathrm{ABC}$ algorithm is proposed for the design of multiplier-less PR MDFT filter banks. The main steps of the algorithm are given below [9 - 12].

\subsection{Initialization}

The filter coefficients of the prototype filter are converted into CSD representation and concatenated to get the initial food source. Since the filter is chosen to have linear phase, the coefficients are symmetric. Hence half the numbers of coefficients only need to be taken. Other food sources can be obtained by randomly perturbing the initial food source. The nectar amount of each food source is calculated and sorted based on its nectar amount. $\mathrm{N}$ vectors with high nectar amount are sent to the next stage [17].

\subsection{Employed bees phase:}

Employed bees will determine a new food source within the neighborhood of the food source in their memory. The new food source near to the current food source is given as $[10,8]$.

$v_{i j}=x_{i j}+\left\lfloor\emptyset_{i j}\left(x_{i j-} x_{k j}\right)\right\rfloor$

where $\emptyset_{i j}$ is a random number between $[-1,1], v_{i j}$ is a candidate food source position, $x_{i}$ is the current food source position, $x_{k}$ is a neighbor food source position and $j \in\{1,2 \ldots . D\}$ is a randomly chosen index which represents a component of each food source position and D is the dimension of the problem. To make sure that the newly generated food sources are within the boundaries of the lookup-table [16], if $v_{i j}<v_{l b}$ then $v_{i j}=v_{l b}$, if $v_{i j}>v_{u b}$ then, $v_{i j}=v_{u b}$ where $v_{l b}$ and $v_{u b}$ are the lower and upper bounds of the look-up-table respectively. If the nectar amount of the new food source is greater than or equal to the current food source, then the old food source will be replaced by the new food source. This mechanism is called greedy selection mechanism.

\subsection{Onlooker bee phase:}

Onlooker bees will get the information from the employed bees regarding the nectar amount of the food source. The onlooker bees will select the food source based on the nectar amount. The probability of the food source located at $\theta_{i}$ chosen by a bee is given as [9].

$P_{i}=\frac{F\left(\theta_{i}\right)}{\sum_{k=1}^{S} F\left(\theta_{k}\right)}$

where $F\left(\theta_{i}\right)$ represents the nectar amount of the food source located at $\theta_{i}$ and $\mathrm{S}$ is the number of food sources around the hive. Number of onlookers is more, for the food sources with high nectar amount. The onlooker bees will search for better food source in the nearby area of the current food source. The onlooker bees will select the new food source if it contains 
high nectar amount and it will reject if the nectar amount is less than the current food source. Greedy selection mechanism is used to select the new food source in this phase.

\subsection{Scout bee phase:}

Each food source has only one employed bee. Hence the number of food sources is same as the number of employed bees. If the nectar amount is not improved even after the predetermined number of trials called 'limit', then the food source is abandoned by its employed bees and they will become scout bees. A scout bee randomly determines the new food source and replaces it with the abandoned one.

\subsection{Termination:}

The algorithm will terminate if the maximum number of iterations is reached, otherwise it will repeat steps 5.2 to 5.4. The optimum filter coefficients will be obtained if the algorithm terminates.

\section{DESIGN OF MULTIPLIER-LESS MDFT FILTER BANK WITH PR}

To design the multiplier-less MDFT filter bank, the first step is to design the FIR prototype filter of the MDFT filter bank with continuous coefficients. Then these coefficients are represented in the CSD space. Since this can degrade the performances of the prototype filter and the MDFT filter bank with PR, optimization technique is to be deployed.

\subsection{Design of continuous coefficient MDFT filter bank}

In the MDFT filter bank, the linear phase prototype FIR filter designed using Parks McClellan method, is complex modulated to derive all the analysis and synthesis filters with linear phase as per equations 1 and 2 . Now our problem is reduced to design a single prototype FIR filter. To achieve PR in the MDFT filter banks, the polyphase realization of the DFT filter banks is used [5]. The phase distortion in MDFT filter banks is zero since all the filters have linear phase. In the design of the MDFT filter banks with PR, aliasing distortion is completely eliminated. Based on the degree of overlap between the adjacent filter responses, some amplitude distortion will occur. In this paper the adjacent filter responses intersect at $3 \mathrm{~dB}$ level, since the passband and stopband edge frequencies are selected carefully.

Design specifications:

$\begin{array}{ll}\text { Maximum Pass-band ripple } & : 0.004 \mathrm{~dB} \\ \text { Minimum Stop-band attenuation } & : 60 \mathrm{~dB} \\ \text { Pass-band edge frequency } & : 0.1220 \pi \\ \text { Stop-band edge frequency } & : 0.12938 \pi \\ \text { Number of channels, M } & : 8\end{array}$

The frequency response plots of the prototype filter and the analysis filters are shown in Fig. 4 and Fig.5 respectively. Fig.6 shows the amplitude distortion function. The aliasing distortion is shown in Fig.7 and the condition for PR is shown in Fig.8. In this example, the condition for aliasing to be zero, given by equation 10 is $2 / \mathrm{M}=0.25$.

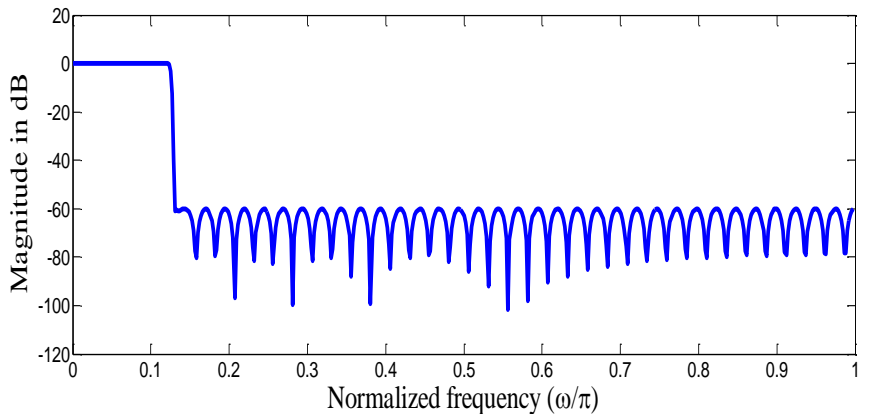

Fig.4. Frequency response of Prototype Filter designed with Continuous Coefficients

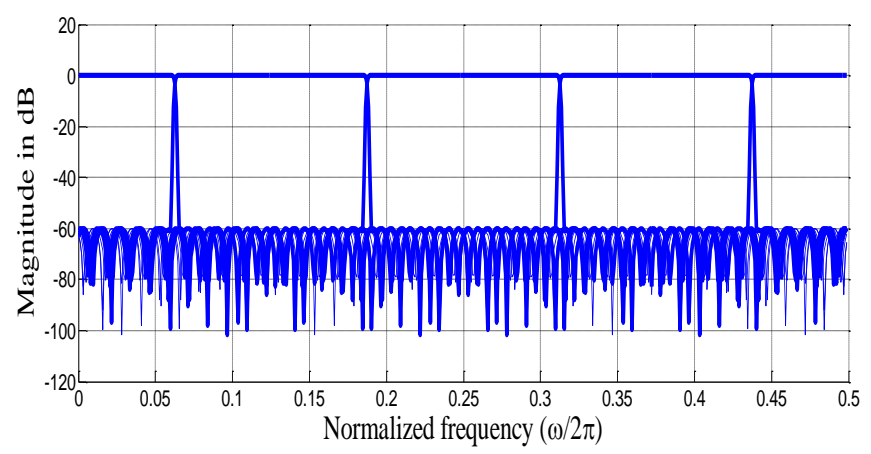

Fig.5. Frequency response of Analysis Filters designed with Continuous Coefficients

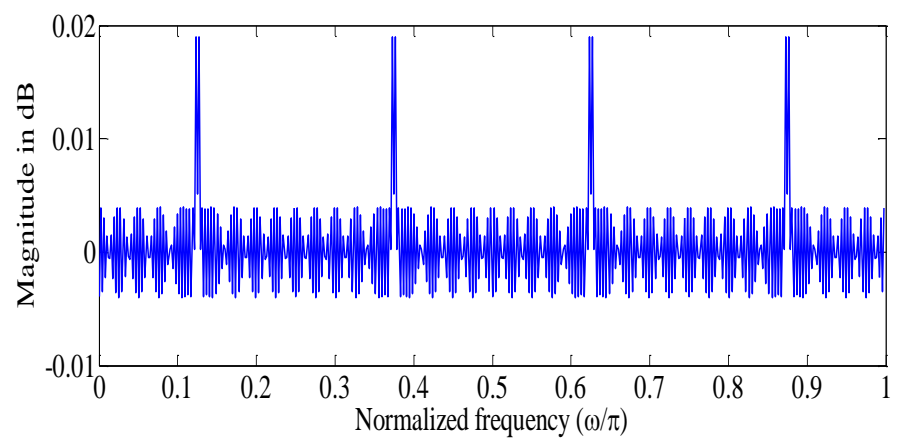

Fig.6. Amplitude distortion function plot (Continuous Coefficients)

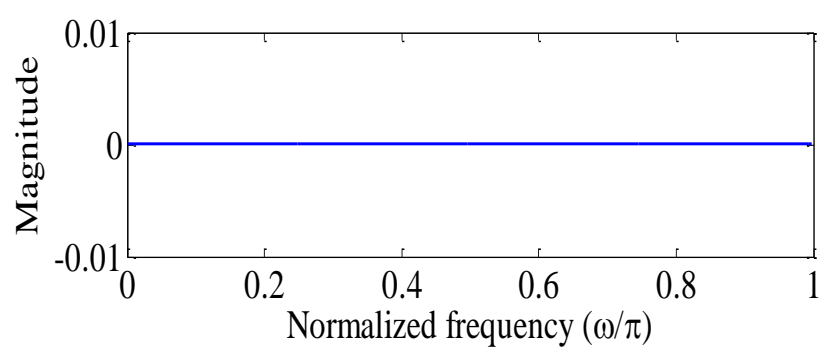

Fig.7. Aliasing distortion function plot of MDFT PR filter bank (Continuous Coefficients) 


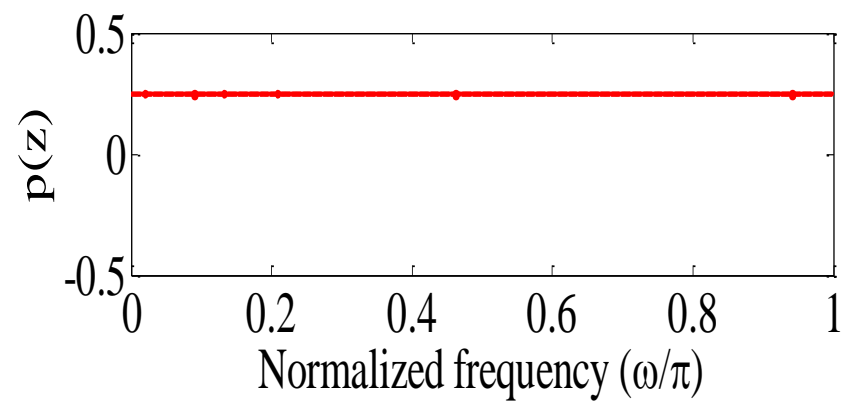

Fig.8. Condition for Perfect Reconstruction

\subsection{Design of the Multiplier-less MDFT filter bank}

The continuous filter coefficients are converted to CSD representation, in order to change the multipliers into adders/subtractor and shifters. A look-up-table is generated to convert the continuous coefficients into CSD representation. Look-up-table as shown in Table. 1 contains the index, 16 bit $\mathrm{CSD}$, its equivalent decimal value and number of non-zero terms in the CSD representation. Table. 2 shows the frequency performance parameters of the continuous coefficients and the maximum precision parameters of the CSD representation for different word lengths used. It is found that the frequency parameters are very close to those with continuous filter coefficients when 18 bit CSD representation with maximum precision is used. But the number of adders due to SPT terms is high as compared to 16 bit CSD representation with maximum precision. Hence some tradeoff is required between the filter response and the complexity. Hence, in this paper, we have chosen 16 bit CSD representation of the coefficients with 3 SPT terms, which leads to the design of MDFT filter banks with less number of adders and without much degradation in the performances of the filter.

The frequency response plot of the prototype filter (CSD rounded coefficients) is shown in Fig.9. The frequency response plot of the analysis filters (CSD rounded coefficients) is shown in Fig.10, the amplitude distortion function (CSD Rounded Coefficients) is shown in Fig.11 and the aliasing distortion is shown in Fig.12. From Table. 3 it is found that the frequency characteristics and amplitude

distortion are degraded with CSD representation, compared to those with continuous coefficients and maximum precision,even though the number of adders is reduced. Hence to improve the frequency parameters and amplitude distortion, an efficient non-linear optimization technique ( $\mathrm{ABC}$ Algorithm) is used here.

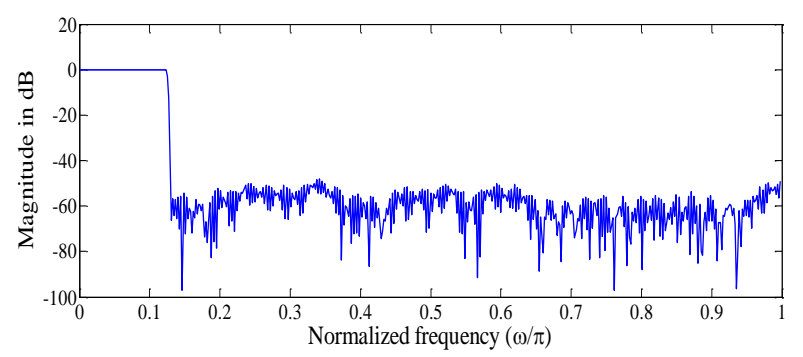

Fig.9. Frequency response of Prototype Filter (CSD Rounded Coefficients)

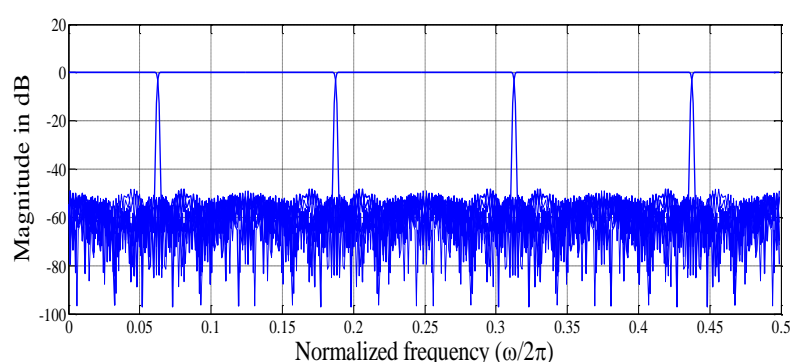

Fig.10. Frequency response of Analysis Filters (CSD Rounded Coefficients)

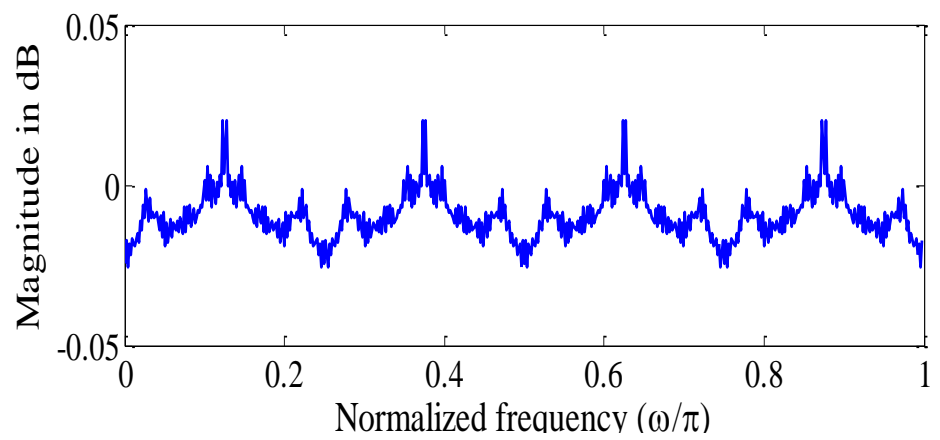

Fig.11. Amplitude distortion function plot of MDFT PR filter bank (CSD Rounded Coefficients)

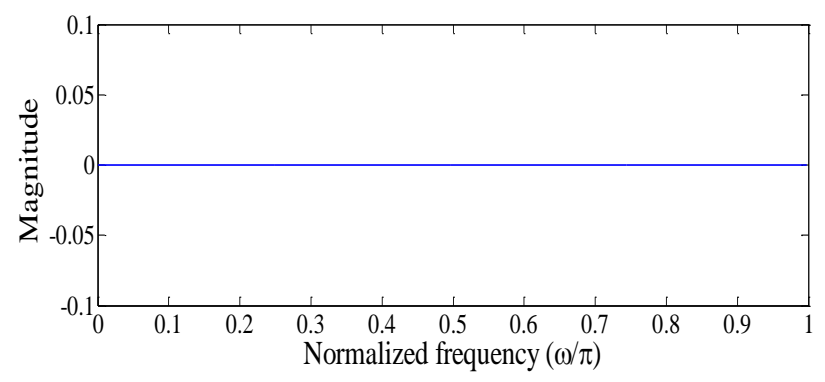

Fig.12. Aliasing distortion function plot of MDFT PR

Table.2. Frequency performances and complexity of the maximum precision CSD represented prototype filter and MDFT filter bank for different word lengths

\begin{tabular}{|c|c|c|c|c|}
\hline & \multicolumn{2}{|c|}{ Prototype filter } & $\begin{array}{c}\text { MDFT } \\
\text { filter bank } \\
\text { (PR) }\end{array}$ & \\
\hline $\begin{array}{c}\text { Word } \\
\text { length }\end{array}$ & $\begin{array}{c}\text { Passband } \\
\text { ripple } \\
(\mathrm{dB})\end{array}$ & $\begin{array}{c}\text { Stopband } \\
\text { attenuation } \\
(\mathrm{dB})\end{array}$ & $\begin{array}{c}\text { Amplitude } \\
\text { distortion } \\
(\mathrm{dB})\end{array}$ & $\begin{array}{c}\text { No. of } \\
\text { adders } \\
\text { due to } \\
\text { SPT }\end{array}$ \\
\hline $\begin{array}{c}\text { Continuous } \\
\text { Coefficients }\end{array}$ & 0.003995 & 60 & 0.01896 & 0 \\
\hline 14 bits & 0.010240 & 51.59 & 0.01077 & 261 \\
\hline 16 bits & 0.005241 & 58.06 & 0.02075 & 493 \\
\hline 18 bits & 0.004676 & 59.38 & 0.01860 & 775 \\
\hline
\end{tabular}




\subsection{Proposed design of the optimal Multiplier-less MDFT filter banks with PR}

The continuous filter coefficients have to be converted into CSD representation and the results should be optimized to design the optimal Multiplierless MDFT filter banks with PR. Hence the objective function for the optimization technique has to be formulated. The prototype FIR filter using Parks McClellan method is used to derive the MDFT filter banks. Hence optimizing the prototype filter coefficients is equivalent to optimizing the MDFT filter banks. The performance degradation due to CSD representation of the continuous filter coefficients is improved by using $\mathrm{ABC}$ optimization algorithm.

\subsubsection{Objective function formulation:}

In the proposed multiplier-less MDFT filter banks with PR, optimized using $\mathrm{ABC}$ algorithm, the aim is to minimize the following objective function.

Let $F_{p}$ be the pass-band ripple, $F_{s}$ the stop-band attenuation, $\omega_{p}$ the pass-band cut-off frequency and $\omega_{s}$ the stop-band cutoff frequency of the prototype low pass filter

$F_{p}=\max _{0<\omega<\omega_{p}}|| H(\omega)|-1|$

$F_{S}=\max _{\omega_{s}<\omega<\pi}|H(\omega)|$

where $H(\omega)$ is frequency response of the prototype filter. The amplitude distortion function of the MDFT filter bank, $T_{\text {dist }}(z)$ is given in equation (11). Hence the error in the amplitude distortion function is given as

$F_{\text {dist }}=\max _{0<\omega<\pi} \mid\left(T_{\text {dist }}(\omega)-1 \mid\right.$

In the CSD represented filter, to reduce the average number of SPT terms, a constraint is added to the objective function. If
$b_{H}$ is the average number of non-zero SPT terms in the filter coefficients and $B_{H}$ is the required upper bound of $b_{H}$, the constraint added to the optimization problem is

$p\left(b_{H}, B_{H}\right)=\max \left(0, b_{H}-B_{H}\right)$

where $B_{H}$ is taken as 3 in this problem.

Hence the optimization problem for the multiplier-less MDFT filter banks with PR is to minimize the objective function $\boldsymbol{\delta}$ which is formulated as

Minimize $\delta=\beta_{1} F_{p}+\beta_{2} F_{S}+\beta_{3} F_{\text {dist }}+\beta_{4} p\left(b_{H}, B_{H}\right)$

where $\beta_{1}, \beta_{2} \beta_{3}$, and $\beta_{4}$ are weights, which define the relative importance given to each term in the objective function. Table. 4 shows the parameters of ABC Algorithm.

The frequency response plot of the prototype filter optimized using $\mathrm{ABC}$ algorithm is shown in Fig.13. The amplitude distortion function optimized using $\mathrm{ABC}$ algorithm is shown in Fig.14. The aliasing distortion is shown in Fig.15. The magnitude responses of the FIR filter before and after using the ABC Algorithm are shown in Figure.16. The linear phase plot of the prototype filter is shown in Fig.17.

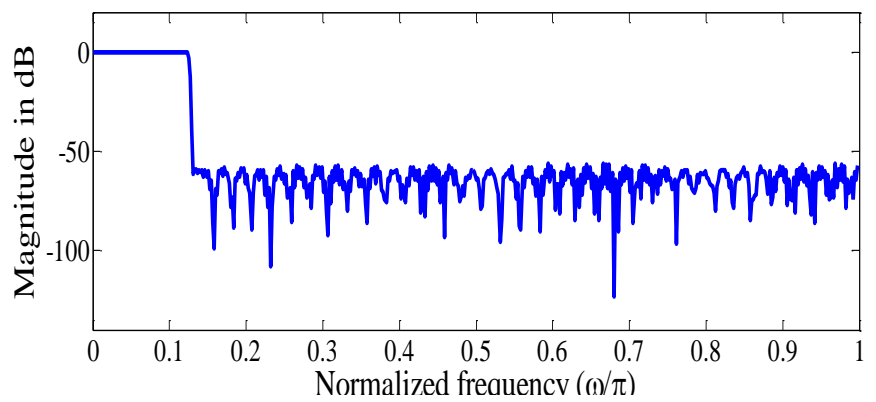

Fig.13. Frequency response of FIR prototype Filter optimized using ABC Algorithm

Table.3. Frequency performance and complexity of the CSD represented prototype filter and MDFT filter bank

\begin{tabular}{|c|c|c|c|c|c|c|}
\hline & \multicolumn{2}{|c|}{ Prototype filter } & MDFT filter bank & \multicolumn{2}{c|}{ Complexity of Implementation } \\
\hline $\begin{array}{c}\text { SPT terms } \\
\text { used }\end{array}$ & $\begin{array}{c}\text { Pass-band } \\
\text { ripple (dB) }\end{array}$ & $\begin{array}{c}\text { Stop-band } \\
\text { attenuation (dB) }\end{array}$ & $\begin{array}{c}\text { Amplitude } \\
\text { distortion (dB) }\end{array}$ & $\begin{array}{c}\text { No. of } \\
\text { structural } \\
\text { adders }\end{array}$ & $\begin{array}{c}\text { Number of } \\
\text { adders due to } \\
\text { SPT terms }\end{array}$ & $\begin{array}{c}\text { Total No. of } \\
\text { adders }\end{array}$ \\
\hline $\begin{array}{c}\text { Maximum } \\
\begin{array}{c}\text { Precision } \\
(8 \text { SPTs })\end{array}\end{array}$ & 0.005241 & 58.06 & 0.02075 & 472 & 493 & 966 \\
\hline $\begin{array}{c}\text { CSD } \\
\text { rounded } \\
(3 \text { SPTs })\end{array}$ & 0.01898 & 48.14 & 0.02551 & 472 & 455 & 928 \\
\hline
\end{tabular}




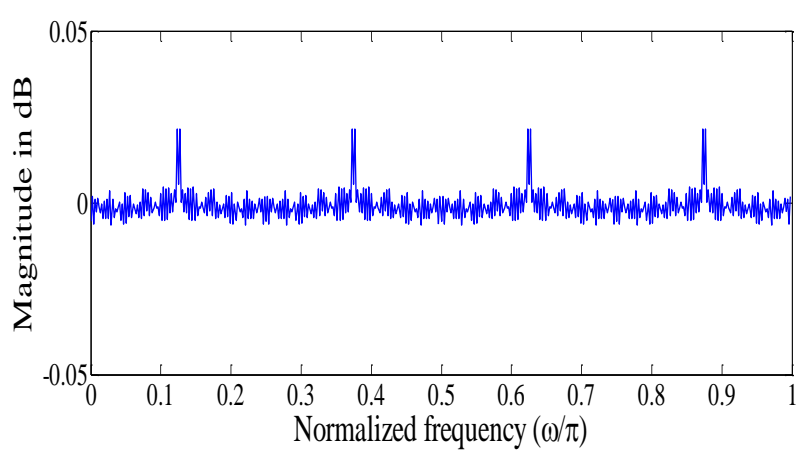

Fig.14. Amplitude distortion function plot of MDFT PR filter bank optimized using ABC Algorithm

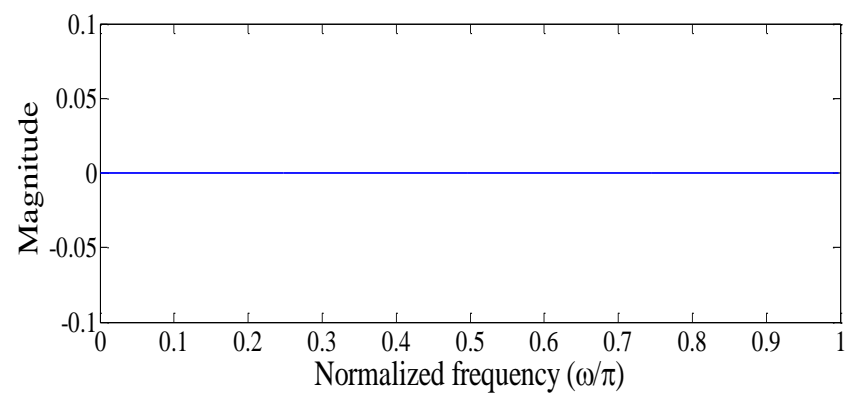

Fig.15. Aliasing distortion function plot using ABC Algorithm

Table.4. Parameters of ABC Algorithm

\begin{tabular}{|c|c|c|}
\hline Population size & Limit cycles & Number of Iterations \\
\hline 50 & 495 & 500 \\
\hline
\end{tabular}

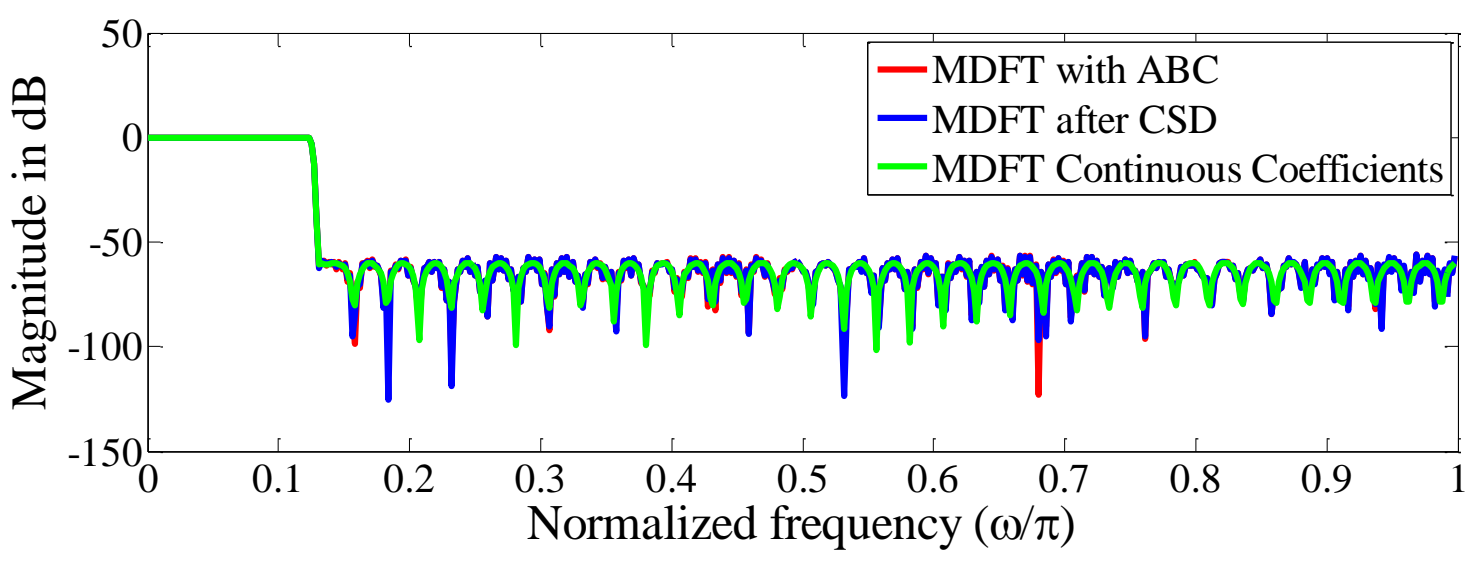

Fig.16. Magnitude response of the FIR prototype filter before and after using the ABC Algorithm

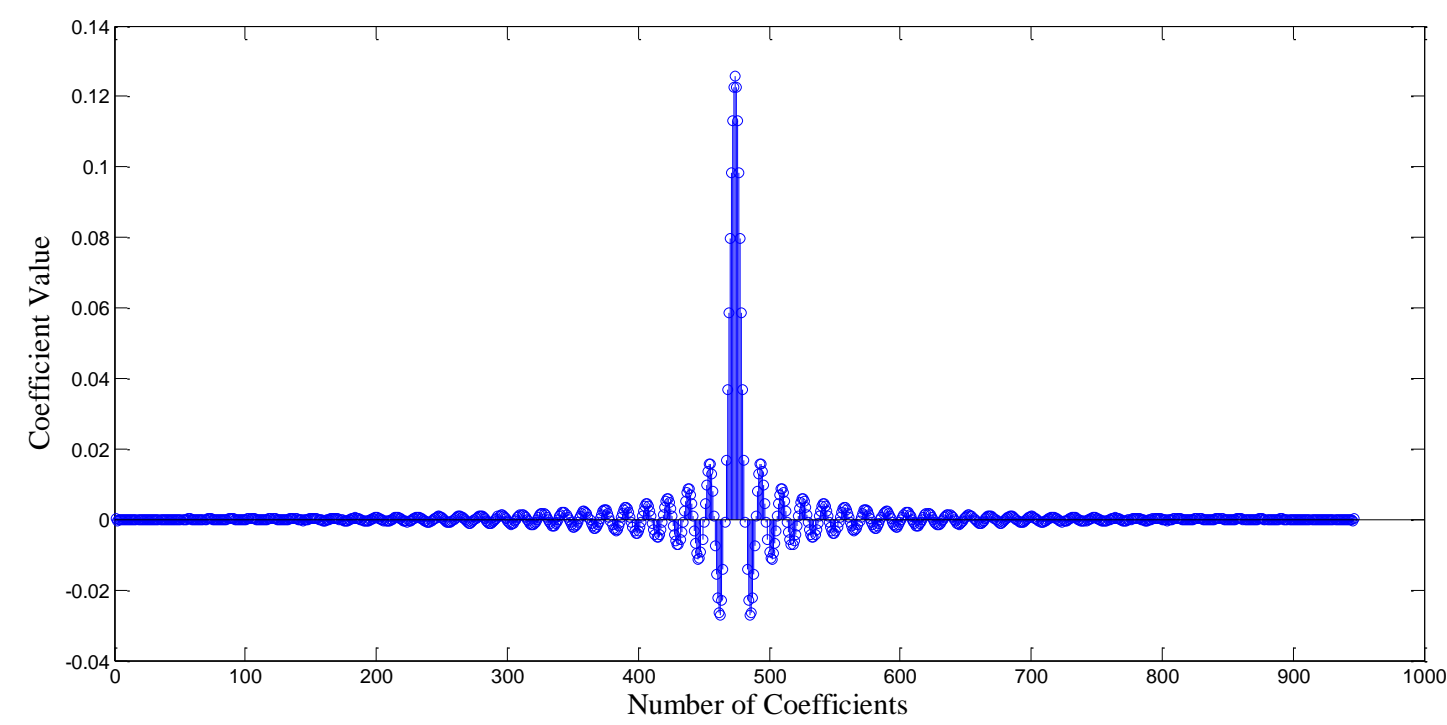

Fig.17. Linear phase plot of prototype filter 
Table.5. Parameter values before and after optimization

\begin{tabular}{|l|c|c|c|}
\hline \multicolumn{1}{|c|}{ Parameters } & $\begin{array}{c}\text { Continuous } \\
\text { Coefficients }\end{array}$ & $\begin{array}{c}\text { CSD rounded } \\
\text { (3 SPTs) } \\
\text { coefficients }\end{array}$ & $\begin{array}{c}\text { ABC } \\
\text { Algorithm }\end{array}$ \\
\hline $\begin{array}{l}\text { Minimum } \\
\text { Passband } \\
\text { ripple(dB) }\end{array}$ & 0.003995 & 0.018980 & 0.005804 \\
\hline $\begin{array}{l}\text { Maximum } \\
\text { Stopband } \\
\text { attenuation(dB) }\end{array}$ & 60.00 & 48.14 & 56.01 \\
\hline $\begin{array}{l}\text { Amplitude } \\
\text { distortion }\end{array}$ & 0.01896 & 0.02551 & 0.02151 \\
\hline $\begin{array}{l}\text { No. of structural } \\
\text { adders }\end{array}$ & 472 & & 472 \\
\hline $\begin{array}{l}\text { No. of adders } \\
\text { due to SPT terms }\end{array}$ & & 0 & 487 \\
\hline $\begin{array}{l}\text { No. of } \\
\text { Multipliers }\end{array}$ & 473 & & 0 \\
\hline
\end{tabular}

\section{RESULTS AND DISCUSSION}

Initially, for the given specifications, an FIR prototype filter with linear phase is designed. The FIR prototype filter coefficients are converted into CSD equivalent representation using a 16 bit look-up-table. To improve the results of the CSD represented filter, ABC optimization algorithm is used. From Table.5, it is found that the optimized filter performances are very close to those with continuous filter coefficients. To get the optimized results we need shifters and adders only. The hardware implementation of the filter bank requires only shifters and adders and is totally multiplier-less. Hence the complexity to implement the MDFT filter bank with PR is reduced. In this paper, all the simulations are performed on an Intel(R) core(TM) i5 processor operating at 2.4 GHz using MATLAB 7.10.0(R2010a).

\section{CONCLUSION}

In this paper, the design of a multiplier-less MDFT with PR using FIR prototype filter is proposed. The continuous filter coefficients are represented in the discrete space using CSD. This degrades the performances. Hence, to improve the performances of the filter bank, a modified ABC algorithm is used.

The proposed design of multiplier-less MDFT filter bank with PR, leads to a very low implementation complexity and hence low power dissipation and low chip area, which are the major requirements in upcoming applications such as software defined radio, wireless communication applications and portable computing systems.

Other metaheuristic optimization techniques such as gravitational search algorithm (GSA), harmony search algorithm (HSA), differential evolution (DE) algorithm and genetic algorithm (GA) can be deployed to design the prototype filter. A comparative study can be made for the performances and implementation complexity using the above algorithms and the best optimization technique can be chosen to design the MDFT PR filter bank with the optimum performance and least implementation complexity.

\section{REFERENCES}

[1] M. G. Bellanger and J.L. Daguet (1974). TDM-FDM transmultiplexer: Digital polyphase and FFT, IEEE Trans. Commun, Vol. COM-22,pp. 1199-1204.

[2] Bindiya T.S. and Elias, E (2014, February).Design of multiplier-less sharp transition width MDFT filter banks using modified metaheuristic algorithms. International Journal of Computer Applications,88(2),1-13.

[3] N. J. Fliege (1993). Computational efficiency of modified DFT - polyphase filter banks. In Proc. $27^{\text {th }}$ Asilomar Conf. Signals ,Systems and Computers, Asilomar Nov.1993,1296-1300.

[4] Fliege, N. J. (1994, April). Modified DFT polyphase SBC filter banks with almost perfect reconstruction. In Acoustics, Speech, and Signal Processing, 1994.ICASSP-94.,1994 IEEE International Conference on, Vol.2, pp.149-152.

[5] Karp, T. and Fliege, N. J. (1999). Modified DFT filter banks with perfect reconstruction. Circuits and Systems II: Analog and Digital Signal Processing, IEEE Transactions on, 46(11), 1404-1414.

[6] Lim, Y. C., Yang, R., Li, D., and Song, J. (1999). Signed power-of-two term allocation scheme for the design of digital filters. Circuits and Systems II: Analog and Digital Signal Processing, IEEE Transactions on, 46(5), 577-584.

[7] Hartely, R. I. (1996). Subexpression sharing in filters using canonic signed multipliers. Circuits and Systems II: Analog and Digital Signal Processing, IEEE Transactions on, 43(10), 677-688.

[8] Bindiya T. S. and Elias, E (2012). Design of Multiplierless Reconfigurable Non-uniform channel filters using metaheuristic algorithms, International Journal of Computer Applications, Published by Foundation of Computer Science, New York, USA, 59(11): 1-11.

[9] D. Karaboga and B. Basturk (2008) . On the performance of artificial bee colony (ABC) algorithm. Applied Soft Computing 8 (2008), 687-697.

[10] Dervis Karaboga , Celel Ozturk, Nurhan Karaboga, Beyza Gorkemli (2012). Artificial bee colony programming for symbolic regression. Erciyes University, Engineering Faculty, Intelligent Systems Research Group. Information Science 209 (2012), 1-15.

[11] Dervis Karaboga (2005). An idea based on Honey bee Swarm for Numerical Optimization. Technical Report TR06, Erciyes University, Engineering Faculty, Computer Engineering Department, 2005.

[12] B. Basturk and D. Karaboga (2006). An artificial bee colony $(\mathrm{ABC})$ algorithm for numeric optimization, in : IEEE Swarm Intelligence Symposium 2006, May 12-14, Indianapolis, IN, USA, 2006.

[13] Fliege, N. J. (1994). Multirate digital signal processing. Chichester, U.K.:Wiley.

[14] R. E. Crochiere and L. R. Rabiner (1983) . Multirate Digital Signal Processing. Englewood Cliffs, NJ: Prentice-Hall. 
[15] P. P. Vaidyanathan (1993) . Multirate systems and filter banks. Englewood Cliffs, NJ: Prentice-Hall.

[16] Manoj, V. J. and Elias, E. (2012). Artificial bee colony algorithm for the design of multiplier-less nonuniform filter bank transmultiplexer. Information Sciences, 192, 193-203.
[17] Manuel, M., and Elias, E. (2013). Design of frequency response masking FIR filter in the canonic signed digit space using modified artificial bee colony algorithm. Engineering Applications of Artificial Intelligence, 26(1), 660-668. 